\title{
Giant increase of critical current density and vortex pinning in Mn doped $\mathrm{K}_{\mathrm{x}} \mathrm{Fe}_{2-\mathrm{y}} \mathrm{Se}_{2}$ single crystals
}

\author{
Mingtao Li, ${ }^{1,2}$ La Chen, ${ }^{1, a)}$ Wen-Long You, ${ }^{3}$ Junyi Ge, ${ }^{4}$ and Jincang Zhang ${ }^{1,2, b)}$ \\ ${ }^{1}$ Department of Physics, Shanghai University, Shanghai 200444, China \\ ${ }^{2}$ Materials Genome Institute, Shanghai University, Shanghai 200444, China \\ ${ }^{3}$ College of Physics, Optoelectronics and Energy, Soochow University, Suzhou 215006, China \\ ${ }^{4}$ INPAC-Institute for Nanoscale Physics and Chemistry, KU Leuven, Celestijnenlaan 200D, B-3001 Leuven, \\ Belgium
}

(Received 11 October 2014; accepted 4 November 2014; published online 12 November 2014)

\begin{abstract}
We report a comparative study of the critical current density $\left(J_{c}\right)$ and vortex pinning among pure and $\mathrm{Mn}$ doped $\mathrm{K}_{\mathrm{x}} \mathrm{Fe}_{2-\mathrm{y}} \mathrm{Se}_{2}$ single crystals. It is found that the $J_{c}$ values can be greatly improved by Mn doping and post-quenching treatment when comparing to pristine pure sample. In contrast to pure samples, an anomalous second magnetization peak (SMP) effect is observed in both $1 \%$ and $2 \% \mathrm{Mn}$ doped samples at $T=3 \mathrm{~K}$ for $H \| a b$ but not for $H \| c$. Referring to Dew-Hughes and Kramer's model, we performed scaling analyses of the vortex pinning force density vs magnetic field in $1 \% \mathrm{Mn}$ doped and quenched pristine crystals. The results show that the normal point defects are the dominant pinning sources, which probably originate from the variations of intercalated $\mathrm{K}$ atoms. We propose that the large nonsuperconducting K-Mn-Se inclusions may contribute to the partial normal surface pinning and give rise to the anomalous SMP effect for $H \| a b$ in Mn doped crystals. These results may facilitate further understanding of the superconductivity and vortex pinning in intercalated iron-selenides superconductors. (C) 2014 AIP Publishing LLC.
\end{abstract}

[http://dx.doi.org/10.1063/1.4901902]

The discovery of iron-based superconductors (SCs) have been stimulating the studies of understanding the mechanism of unconventional high- $T_{c}$ superconductivity, ${ }^{1,2}$ and the issues on their potential applications are also inspired simultaneously. ${ }^{3}$ Among iron-based superconducting families, ${ }^{2}$ the so called " 122 " iron-based $\mathrm{SCs}$ with $\mathrm{ThCr}_{2} \mathrm{Si}_{2}$ structure are particularly attractive and promising for future high field applications due to their high $T_{c}$ (up to $38 \mathrm{~K}$ ), upper critical field $\left(\mu_{0} H_{c 2} \sim 100 \mathrm{~T}\right)$, low anisotropy $(\Gamma \sim 2)$, and capacity of carrying large critical current density $\left(J_{c} \sim 10^{6} \mathrm{~A} / \mathrm{cm}^{2}\right){ }^{4-7}$ Sharing a similar crystal structure with " 122 " SCs, the mono-ion intercalated $A_{\mathrm{X}} \mathrm{Fe}_{2-\mathrm{y}} \mathrm{Se}_{2}(A=\mathrm{K}, \mathrm{Rb}, \mathrm{Cs}, \mathrm{Tl}) \mathrm{SCs}$ with $T_{c} \sim 30 \mathrm{~K}$ have received considerable attention because of their intriguing properties. ${ }^{8}$ Very high $\mu_{0} H_{c 2}$ values ( $>50 \mathrm{~T}$ at $T=0 \mathrm{~K}$ ) have been revealed by the high field magnetotransports and radiofrequency (rf) contactless penetration depth measurements on $\mathrm{K}_{\mathrm{x}} \mathrm{Fe}_{2-\mathrm{y}} \mathrm{Se}_{2}$ and $(\mathrm{Tl}, \mathrm{Rb})_{\mathrm{x}} \mathrm{Fe}_{2-\mathrm{y}} \mathrm{Se}_{2}$ single crystals. ${ }^{9-12}$ More importantly, the arsenic-free SCs have lower toxicity than iron-arsenic SCs. To this end, a question is naturally raised whether the $A_{\mathrm{x}} \mathrm{Fe}_{2-\mathrm{y}} \mathrm{Se}_{2} \mathrm{SCs}$ could be a candidate material for high field magnet. However, the bulk $A_{\mathrm{x}} \mathrm{Fe}_{2-\mathrm{y}} \mathrm{Se}_{2}$ single crystals are always infested by the intrinsic phase separation, ${ }^{13-16}$ which is a big obstacle to practical applications. Recent neutron diffraction studies demonstrate that the insulating phase has a chemical formula $\mathrm{K}_{2} \mathrm{Fe}_{4} \mathrm{Se}_{5}$ forming a $\sqrt{5} \times \sqrt{5}$ iron vacancy ordered block antiferromagnetic (AF) structure at low temperature while the superconducting phase arises from

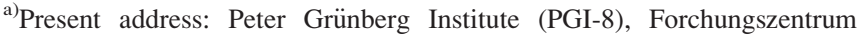
Jülich, D-52425 Jülich, Germany.

b) Author to whom correspondence should be addressed. Electronic mail: jczhang@staff.shu.edu.cn
}

alkali deficient $\mathrm{K}_{\mathrm{z}} \mathrm{Fe}_{2} \mathrm{Se}_{2}$ ( $\mathrm{ThCr}_{2} \mathrm{Si}_{2}$ structure), which is embedded in the matrix of the insulating block AF phase. ${ }^{16}$

Different schemes, such as high temperature melt (within one or two steps), ${ }^{14,15,17-20}$ Bridgman method, ${ }^{21}$ and optical floating-zone technique, ${ }^{22}$ have been employed to grow single crystalline $A_{\mathrm{x}} \mathrm{Fe}_{2-\mathrm{y}} \mathrm{Se}_{2}$. Lei and Petrovic show that pristine $\mathrm{K}_{\mathrm{x}} \mathrm{Fe}_{2-\mathrm{y}} \mathrm{Se}_{2}$ single crystals can only carry small $J_{c}$ values. ${ }^{17}$ It was reported that the $J_{c}$ values could be improved by quenching or Te/S doping, ${ }^{14,15,20-24}$ but still one or two orders smaller than " 11 " iron-chalcogenide and "122" iron-arsenic SCs. ${ }^{4-7,25,26}$ To improve the $J_{c}$ and evaluate the high field performance of $A_{\mathrm{x}} \mathrm{Fe}_{2-\mathrm{y}} \mathrm{Se}_{2} \mathrm{SCs}$, the study of vortex pinning mechanism needs to be carried out especially on high quality samples. So far, there are only few investigations on the vortex pinning in "122"-like $\mathrm{K}_{\mathrm{x}} \mathrm{Fe}_{2} \mathrm{Se}_{2}$ SCs. ${ }^{19,23,24}$ Recently, we reported that the phase separation and the superconductivity of $\mathrm{K}_{\mathrm{x}} \mathrm{Fe}_{2-\mathrm{y}} \mathrm{Se}_{2}$ can be significantly enhanced by slight Mn dopant. ${ }^{18,19}$ More interestingly, it is demonstrated by ac susceptibility measurements that the $1 \%$ Mn doped single crystal has very strong intrinsic pinning, ${ }^{19}$ of which the derived activation energy $U_{0}$ shows nearly isotropic behavior for parallel $(H \| a b)$ and perpendicular $(H \| c)$ to $\mathrm{Fe}-\mathrm{Se}$ plane and reaches high values of $10^{5} \mathrm{~K}$ at low fields.

To further elucidate the origin of the strong pinning induced by Mn doping, here we present a comparative study of the magnetic vortex pinning properties for pure and $\mathrm{Mn}$ doped $\mathrm{K}_{\mathrm{x}} \mathrm{Fe}_{2-\mathrm{y}} \mathrm{Se}_{2}$ single crystals. The magnetic hysteresis loops (MHLs) have been measured to extract the $J_{c}$ values. We will discuss the vortex pinning mechanism from the analysis of pinning force density $(F)$ vs magnetic fields $\left(\mu_{0} H\right)$ in the framework of Dew-Hughes and Kramer's model. 
The single crystals of $\mathrm{Mn}$ doped $\mathrm{K}_{0.8} \mathrm{Fe}_{2-z} \mathrm{Mn}_{z} \mathrm{Se}_{2}$ $(\mathrm{z}=0,0.01,0.02$, and 0.03$)$ were grown from the melt of (Fe,Mn)Se precursor and $\mathrm{K}$ metals. ${ }^{18,19}$ The as-grown undoped crystal was annealed and quenched into an ice water bath to improve the superconductivity. ${ }^{14,15,18}$ Energy dispersive $\mathrm{x}$-ray (EDX) spectroscopy has been carried out for composition analysis. ${ }^{18}$ The mean compositions of the four samples were determined to be $\mathrm{K}_{0.76} \mathrm{Fe}_{1.89} \mathrm{Se}_{2}$ (S1), $\mathrm{K}_{0.79} \mathrm{Fe}_{1.88} \mathrm{Se}_{2}$ (S1-quenched), $\mathrm{K}_{0.80} \mathrm{Fe}_{1.84} \mathrm{Mn}_{0.012} \mathrm{Se}_{2}$ (S2), $\mathrm{K}_{0.80} \mathrm{Fe}_{1.86} \mathrm{Mn}_{0.020} \mathrm{Se}_{2}(\mathrm{~S} 3)$, and $\mathrm{K}_{0.73} \mathrm{Fe}_{1.87} \mathrm{Mn}_{0.024} \mathrm{Se}_{2}$ (S4) from nine points of EDX spectrum. The MHLs up to $\pm 7 \mathrm{~T}$ with $H \| a b$ and $H \| c$ were measured using a vibrating sample magnetometer (VSM).

Figures 1(a) and 1(b) show the MHLs with $H \| c$ and $H \| a b$ at $T=3 \mathrm{~K}$ for the four samples, respectively. The MHLs of lightly Mn-doped samples become much bigger and more symmetric than those of the pristine pure samples, in accord with the observation of enhanced superconductivity by Mn dopant. ${ }^{18}$ However, the area of MHLs becomes smaller with an increasing Mn doping content from $\mathrm{z}=0.01$ to 0.03 . There is a most pronounced irreversibility of MHLs in S2 sample for both $H \| c$ and $H \| a b$, and a strong vortex pinning therein was previously suggested based on the analysis of activation energy $U_{0}$ of S2 sample. ${ }^{19}$ By contrast to the findings in " 122 " iron-arsenic SCs, ${ }^{4,5}$ the zero-field central peak (ZFCP) for $H \| c$ is not observed in both S2 and S3 samples. The absence of ZFCP was also reported in high quality $\mathrm{K}_{\mathrm{x}} \mathrm{Fe}_{2-\mathrm{y}} \mathrm{Se}_{2}$ and $\mathrm{Rb}_{\mathrm{x}} \mathrm{Fe}_{2-\mathrm{y}} \mathrm{Se}_{2}$ single crystals. ${ }^{20,21,27}$ The feature has been attributed to the intrinsic percolative superconductivity due to the intrinsic phase separation. ${ }^{13,27}$ Intriguingly, besides the appearance of ZFCP for $H \| a b$, a second magnetization peak (SMP) effect (also called fishtail effect) presents in $\mathrm{S} 2$ and $\mathrm{S} 3$ samples at $T=3 \mathrm{~K}$, implying the vortex pinning is abnormally enhanced with increasing the magnetic field. It is noted both ZFCP and SMP effects have been observed in MHLs of S-doped $\mathrm{K}_{\mathrm{x}} \mathrm{Fe}_{2-\mathrm{y}} \mathrm{Se}_{2}$ single
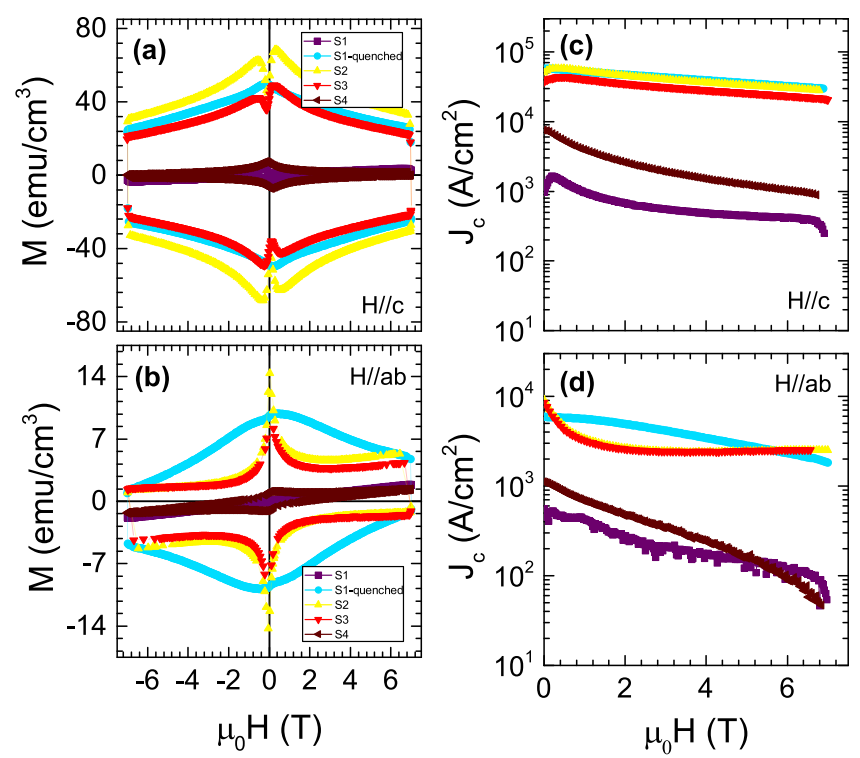

FIG. 1. Isothermal MHLs at $T=3 \mathrm{~K}$ for S1, S1-quenched, S2, S3, and S4 samples. (a) $H \| c$ and (b) $H \| a b$. The critical current density at $T=3 \mathrm{~K}$ as a function of magnetic field for S1, S1-quenched, S2, S3, and S4 samples. (c) $H \| c$ and (d) $H \| a b$. crystal (only for $H \| c$ ) ${ }^{23}$ " 11 " iron-chalcogenide and " 122 " iron-arsenic SCs. ${ }^{4-7,25,26}$ Moreover, after annealing and quenching the crystals at around $673 \mathrm{~K},,^{14,15,18,24}$ the superconducting properties of pristine sample can be enormously improved, as comparable to the Mn doped samples. Here, the symmetric features of MHLs in S1-quenched samples are similar to those reported, ${ }^{14,15,24}$ suggesting that the dominant pinning sources are from bulk instead of surface barriers after thermal treatment. This allows one to make a further comparative study on the vortex pinning between the $\mathrm{S} 2$ and S1-quenched samples.

To estimate the $J_{c}\left(\mu_{0} H\right)$ values from MHLs curves, we employ the extended Bean critical state model. ${ }^{28}$ Accordingly, the field dependence of in-plane $J_{c}^{a b}(H \| c)$ for a rectangular shape is given by the relation

$$
J_{c}^{a b}\left(\mu_{0} H\right)=20 \Delta M /[w(1-w / 3 l)],
$$

where $w$ and $l$ are the width and length of the sample and $\Delta M$ is the difference between the magnetization values for increasing and decreasing field. Here, the thickness $(t)$ of the sample is set as $t<w<l$ and the magnetization is measured in the unit of emu $/ \mathrm{cm}^{3}$. Nevertheless, the evaluation of $J_{c}$ becomes more complex for $H \| a b$. There are two different contributions to the supercurrent, namely, the one parallel to the Fe-Se layers $\left(J_{c}^{a b^{*}}\right.$, usually $\left.J_{c}^{a b^{*}} \neq J_{c}^{a b}\right)$ and the other perpendicular to the $\mathrm{Fe}$-Se layers $\left(J_{c}^{c}\right)$. In the limit of $l, w \gg t J_{c}^{a b^{*}} / 3 J_{c}^{c}$, the inter-plane $J_{c}^{c}$ is approximately given by $J_{c}^{c}=20 \Delta M / t$. The calculated $J_{c}^{a b}$ and $J_{c}^{c}$ are shown in Fig. 1(c) and 1(d). The highest $J_{c}(0)$ of S2 sample at $T=3 \mathrm{~K}$ is extracted to be $J_{c}^{a b}(0)=5.8 \times 10^{4} \mathrm{~A} / \mathrm{cm}^{2}$ and $J_{c}^{c}(0)=9.2 \times 10^{3} \mathrm{~A} / \mathrm{cm}^{2}$ for $H \| c$ and $H \| a b$, respectively. With further increasing the field, the $J_{c}$ shows only weak field dependence for $H \| c$ while first decreases but increases again at $\mu_{0} H=3.6 \mathrm{~T}$ for $H \| a b$ in both S2 and S3 samples. These results indicate that the Mn doping can be an effective way to improve the performance of capability for carrying supercurrents at high fields in $\mathrm{K}_{\mathrm{x}} \mathrm{Fe}_{2-\mathrm{y}} \mathrm{Se}_{2} \mathrm{SCs}$. However, the obtained $J_{c}\left(\mu_{0} H\right)$ values are still one or two orders smaller than the previous results for " 11 " iron-chalcogenide and "122" iron-arsenic SCs. ${ }^{4,75,26}$ Comparing to pristine $\mathrm{K}_{\mathrm{x}} \mathrm{Fe}_{2-\mathrm{y}} \mathrm{Se}_{2}$ single crystals, the $J_{c}(0)$ values of $\mathrm{S} 2, \mathrm{~S} 3$, and S1-quenched samples are much larger than $\mathrm{S} 1$ sample $\left(J_{c}^{a b}(0)=5.6 \times 10^{2} \mathrm{~A} / \mathrm{cm}^{2}\right.$ and $J_{c}^{c}(0)=7.5 \times 10^{2} \mathrm{~A} / \mathrm{cm}^{2}$ at $T=3 \mathrm{~K})$ and previously reported results, ${ }^{17}$ implying that both Mn doping and post-quenching can improve $J_{c}\left(\mu_{0} H\right)$. For S1-quenched, S2, and S3 samples, the $J_{c}\left(\mu_{0} H\right)$ values at low field are comparable to the reported results of the thermal quenched $\mathrm{K}_{\mathrm{x}} \mathrm{Fe}_{2-\mathrm{y}} \mathrm{Se}_{2},{ }^{14,15,23}$ one-step grown $\mathrm{K}_{\mathrm{x}} \mathrm{Fe}_{2-\mathrm{y}} \mathrm{Se}_{2},{ }^{20}$ and Bridgman-method grown $\mathrm{Rb}_{\mathrm{x}} \mathrm{Fe}_{2-\mathrm{y}} \mathrm{Se}_{2}$ single crystals. ${ }^{21}$ It is also noted that $J_{c}^{a b}\left(J_{c}^{c}\right)$ of Mn doped samples is larger than the S-doped $\mathrm{K}_{\mathrm{x}} \mathrm{Fe}_{2-\mathrm{y}} \mathrm{Se}_{2}$ single crystals with a SMP effect for $H \| c .^{24}$ In contrary to other " 122 " systems, ${ }^{4,5}$ the SMP effect disappears quickly for S2/S3 samples with an increasing temperature. ${ }^{29}$

We now turn to discuss the mechanism of vortex pinning in S1-quenched and S2 samples. The magnetic field dependence of the pinning force density defined by $F=\left|\mu_{0} \boldsymbol{H} \times \boldsymbol{J}\right|$ has been investigated in the frame of single vortex pinning regime (i.e., neglecting the intervortex 
interactions), ${ }^{30}$ the pinning force of type-II superconductors can be scaled as

$$
f=F / F_{\text {max }} \propto h^{p}(1-h)^{q},
$$

where $F_{\max }$ is the maximum pinning force density and $h=H / H_{\text {irr }}$ is the ratio between external field $\mu_{0} H$ and irreversibility field $\mu_{0} H_{i r r}$. Based on $J_{c}-\mu_{0} H$ data, $\mu_{0} H_{i r r}$ can usually be determined as the extrapolated zero $J_{c}$ value from Kramer plot (noted by criterion I), ${ }^{31}$ i.e., $J_{c}^{1 / 2}\left(\mu_{0} H\right)^{1 / 4}$ is plotted as a function of $\mu_{0} H$. Nevertheless, if the pinning is dominated by point defects with their dimensions smaller than the vortex lattice parameter (given by $a_{0}=\left(2 \Phi_{0} / \sqrt{3} \mu_{0} H\right)^{1 / 2}, \Phi_{0}$ is flux quantum), plot of $J_{c}^{1 / 2} \propto(1-h)$ will be expected and the determination of $\mu_{0} H_{\text {irr }}$ can be done by linearly extrapolating down to zero of $J_{c}^{1 / 2}-\mu_{0} H$ curves (noted by criterion II). ${ }^{30}$ As shown in Figs. 2(a) and 3(a), it is found that the Kramer plot is more appropriate for S2 sample while the Dew-Hughes plot is suitable to S1-quenched sample. This indicates that the point pinning centers play major roles in S1quenched sample. Moreover, the determined $\mu_{0} H_{i r r}$ as a
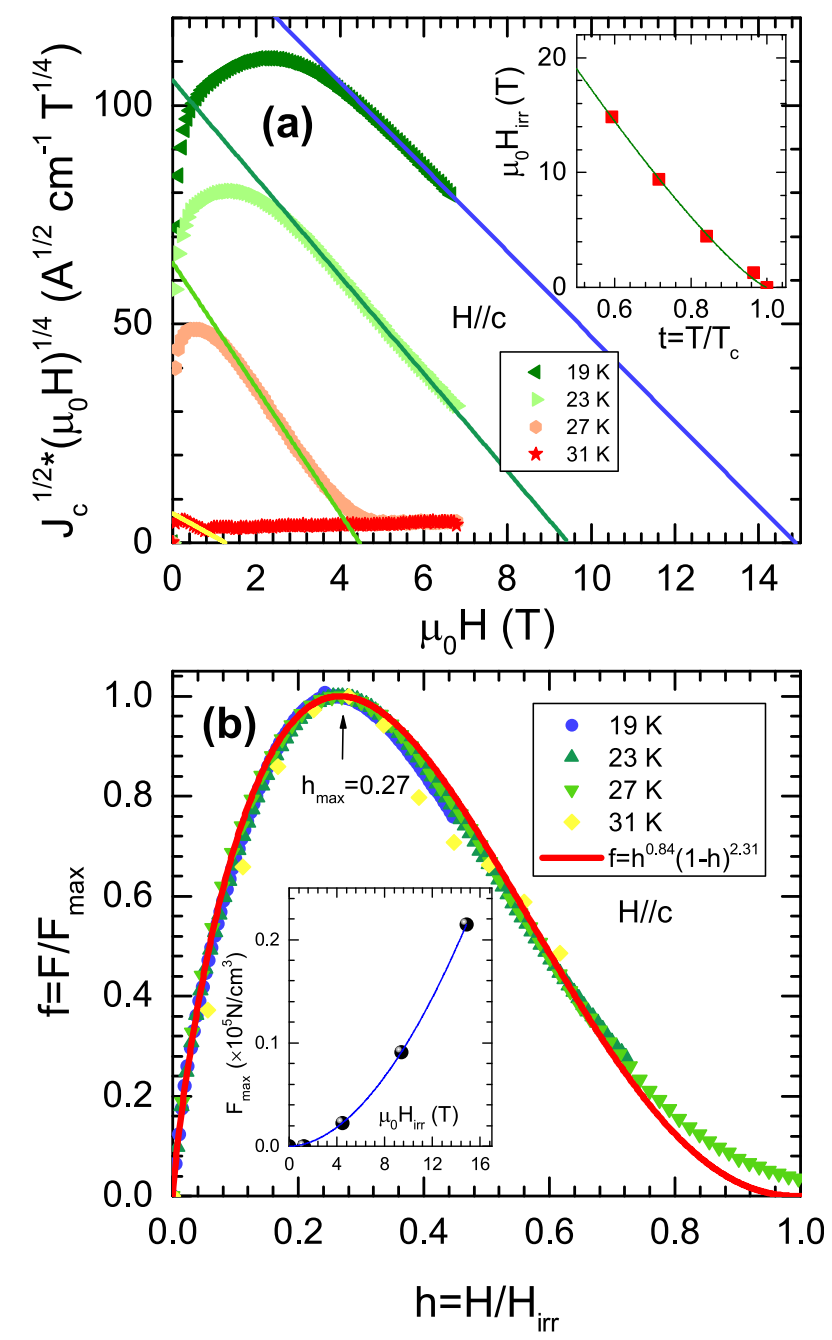

FIG. 2. (a) Kramer plot of $J_{c}^{1 / 2}\left(\mu_{0} H\right)^{1 / 4} \propto \mu_{0} H$ obtained at $T=19,23,27$, $31 \mathrm{~K}$. Solid lines are linear fit curves. Inset shows the reduced temperature dependence of irreversible field with solid line as the fit of formula $\mu_{0} H_{\text {irr }} \propto$ $(1-t)^{n}$ with $n=1.23$. (b) The $f(h)$ data points at various temperatures. Solid line is the fit curve using scaling form $f \propto h^{p}(1-h)^{q}$ with $p=0.84$ and $q=2.31$. Inset shows plot of $F_{\max } \propto\left(\mu_{0} H_{i r r}\right)^{m}$ with $m=1.87$.
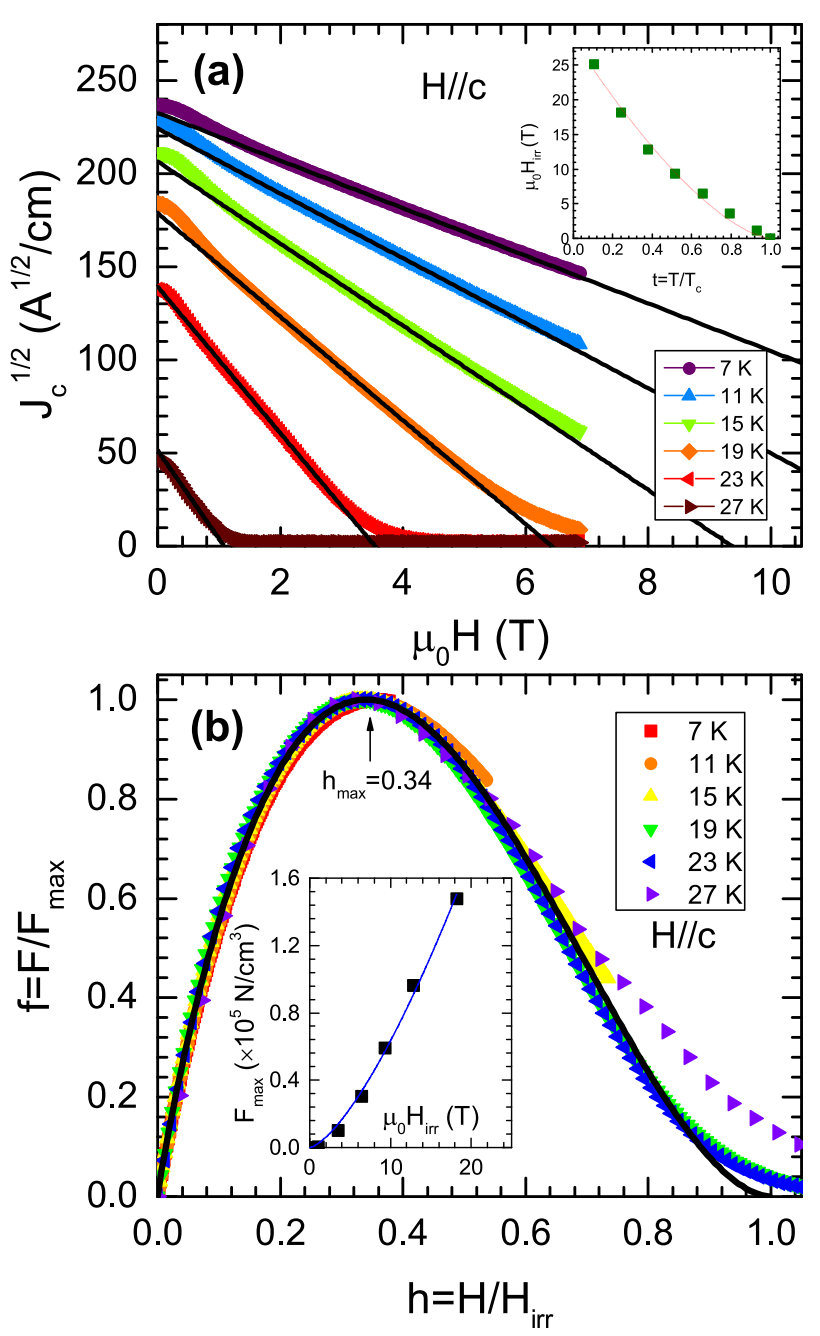

FIG. 3. (a) Plot of $J_{c}^{1 / 2} \propto \mu_{0} H$ obtained at $T=7,19,23,27$, and $31 \mathrm{~K}$. Solid lines are linear fit curves. Inset shows the reduced temperature dependence of irreversible field with solid line as the fit of formula $\mu_{0} H_{\text {irr }} \propto(1-t)^{n}$ with $n=1.52$. (b) The reduced field dependence of normalized flux pinning force at various temperatures. Solid line is the fit curve using scaling form $f \propto h^{p}(1-h)^{q}$ with $p=0.94$ and $q=1.83$. Inset shows plot of $F_{\max } \propto$ $\left(\mu_{0} H_{\text {irr }}\right)^{m}$ with $m=1.45$.

function of reduced temperature $\left(t=T / T_{c}\right)$ can be fitted by empirical formula $\mu_{0} H_{i r r}(T)=\mu_{0} H_{i r r}(0)(1-t)^{n} \quad$ with $n=1.23$ for $\mathrm{S} 2$ and $n=1.52$ for $\mathrm{S} 1$-quenched samples, which are quite close to the typical value $n=1.50$ for threedimensional giant flux creep. ${ }^{32}$ It is also noted that the fitted exponents are in agreement with that $(n=1.49$ for $H \| c)$ determined by ac susceptibility measurements on S2 sample. ${ }^{19}$

To further explore the vortex pinning regime in $\mathrm{S} 2$ and $\mathrm{S} 1$-quenched samples, the temperature and field dependence of the vortex pinning force density are plotted in Figs. 2(b) and $3(\mathrm{~b})$. It is found that the normalized $f(h)$ curves follow a temperature independent scaling law, indicating there is one dominant pinning mechanism for both samples. Fitting the data by Eq. (2), we obtained $p=0.84$ and $q=2.31$ with peak position $h_{\max }=p / p+q=0.27$ for S2 while $p=0.94$ and $q=1.83$ with $h_{\max }=0.34$ for $\mathrm{S} 1$-quenched sample. According to Dew-Hughes' model, ${ }^{30}$ it is expected that the peak position occurs at $h_{\max }=0.33$ with $p=1$ and $q=2$ for normal point pinning centers while $h_{\max }=0.20$ with $p=0.5$ and $q=2$ for normal surface pinning centers (e.g., grain boundaries) with 
core interactions. For the latter case, Kramer also predicted the same scaling expression for the shear breaking in the presence of a set of planar pinning centers. ${ }^{31}$ The fact, that $h_{\max }$ of S1-quenched sample is very close to the theoretical expectation, indicates that its dominant pinning sources are the normal point pinning centers, consistent with the previous finding on quenched $\mathrm{K}_{\mathrm{x}} \mathrm{Fe}_{2-\mathrm{y}} \mathrm{Se}_{2}$ crystal. $^{23}$ Similar peak position was also observed in doped $\mathrm{BaFe}_{2} \mathrm{As}_{2}$ SCs. ${ }^{4,5}$ However, the peak position is much lower than $h_{\max }=0.33$ for S2 sample, implying that the normal surface pinning centers may also contribute to the vortex pinning. Similar scaling with different $h_{\max }$ positions (i.e., $h_{\max }=0.27$ by criterion I for sample A, $h_{\max }=0.30$ by criterion I or $h_{\max }=0.33$ by criterion II for sample B) was also discovered in two batches of $\mathrm{FeTe}_{0.7} \mathrm{Se}_{0.3}$ single crystals. ${ }^{26}$

Furthermore, it is important to shed light on the potential pinning centers preserved in S2 and S1-quenched crystals. Recent XRD, ${ }^{18}$ SEM, and TEM experiments ${ }^{33,34}$ on Mn doped samples show that the Mn atoms can hardly enter the superconducting stripes phase but form nonsuperconducting $\mathrm{K}-\mathrm{Fe}-\mathrm{Mn}$ inclusion phases, which are embedded in superconducting stripes. ${ }^{33}$ Analogous to the role of second phase in high- $T_{c}$ cuprates, ${ }^{35}$ these large normal inclusions are probably the pinning sources for surface pinning regime in $\mathrm{Mn}$ doped $\mathrm{K}_{\mathrm{X}} \mathrm{Fe}_{2-\mathrm{y}} \mathrm{Se}_{2}$ single crystals, since their dimensions are larger than the coherence length $\xi$ and may give rise to the SMP effect. This leads to the conjecture that the vortex pinning induced by these normal inclusions rather than twin boundaries may be responsible for the observation of strong plastic pinning in S2 sample. ${ }^{19}$ On the other hand, the variations of $\mathrm{K}$ atoms ${ }^{6,36}$ probably serve as the major normal point pinning centers in $\mathrm{K}_{\mathrm{z}} \mathrm{Fe}_{2} \mathrm{Se}_{2}$ even though the lattice defects, e.g., $\mathrm{Fe} / \mathrm{Se}$ vacancies cannot be totally excluded. ${ }^{15,16,37,38}$ As shown in the insets of Fig. 2(b) and Fig. 3(b), the $F_{\text {max }}$ obeys a scaling form of $F_{\text {max }} \propto\left(\mu_{0} H_{\text {irr }}\right)^{m}$ with $m=1.87$ and $m=1.45$ for S2 and S1-quenched samples, respectively. These values are somehow smaller than theoretical value $n=2$, which may result from the uncertainty of determination of $\mu_{0} H_{\text {irr }}$ values by the concerned criteria. Lei and Petrovic reported a scaling exponent $n=1.67$ for their quenched $\mathrm{K}_{\mathrm{x}} \mathrm{Fe}_{2-\mathrm{y}} \mathrm{Se}_{2}$ crystal, ${ }^{23}$ in which the normal point pinning regime becomes dominant above $10 \mathrm{~K}$.

As mentioned before, even though $f=F / F_{\text {max }}$ often scales monotonically with $h=H / H_{\text {irr }}$, it is difficult to determine $\mu_{0} H_{\text {irr }}$ accurately. ${ }^{26,35,39}$ To lessen the uncertainty in the pinning analysis, the $F(H)$ data were rescaled by $h^{*}=$ $H / H_{\max }\left(\mu_{0} H_{\max }\right.$ is the magnetic field corresponding to the maximum of $F$ ) as widely adopted in high- $T_{c}$ cuprates $\mathrm{SCs},{ }^{35,39}$ and then the scaling of $f^{*}(h)$ data can be given by the following equations: ${ }^{35,39}$

$$
\begin{gathered}
f^{*}=3 h^{2}(1-2 h / 3) \text { for } \Delta \kappa \text { pinning, } \\
f^{*}=\frac{9}{4} h(1-h / 3)^{2} \text { for normal point pinning, } \\
f^{*}=\frac{25}{16} h^{1 / 2}(1-h / 5)^{2} \text { for surface pinning. }
\end{gathered}
$$

Shown in Figs. 4(a) and 4(b) are plots of the new scaled data for S2 and S1-quenched samples, respectively. It turns out that the experimental data follow Eq. (4) well in wider field

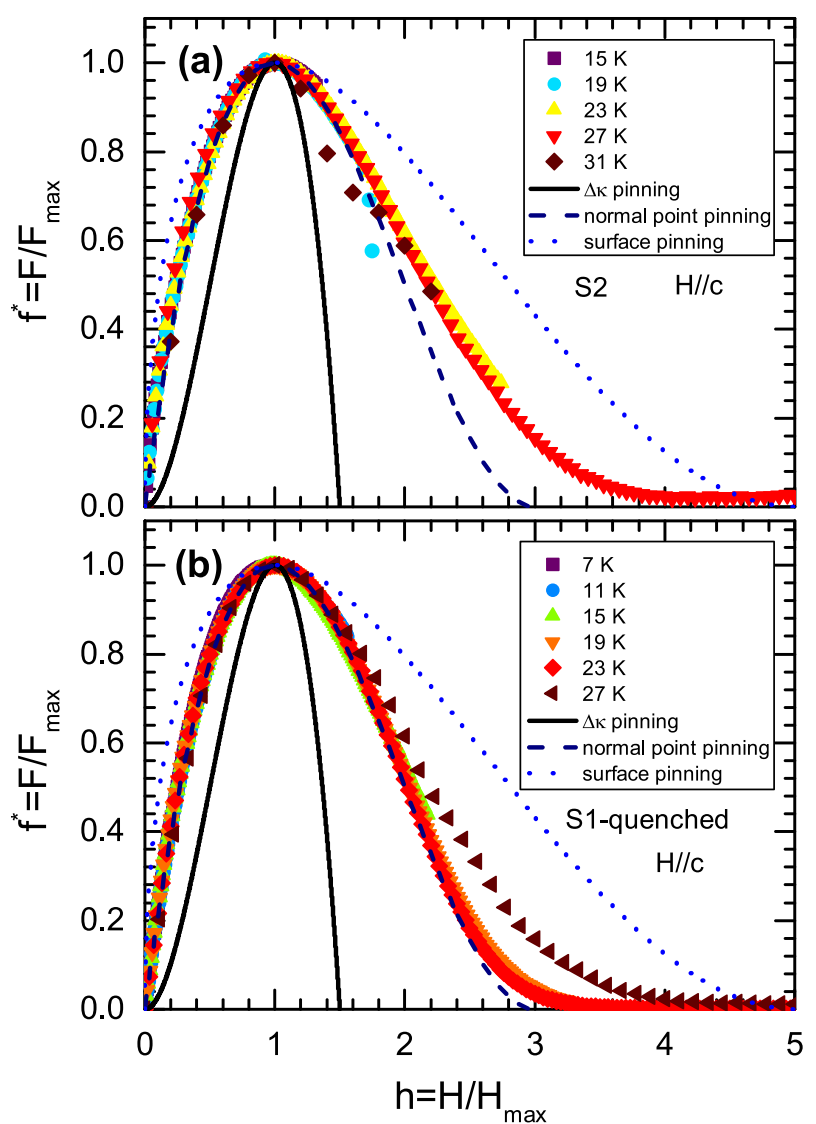

FIG. 4. Plots of $f^{*}(h)$ at different temperatures for (a) S2 sample, (b) S1quenched sample. Solid line is the fit curve of Eq. (3) for $\Delta \kappa$ pinning. Dashed line is the fit curve of Eq. (4) for normal point-pinning. Dotted line is the fit curve of Eq. (5) for surface pinning.

range for $\mathrm{S} 1$-quenched than $\mathrm{S} 2$ sample, confirming the actual dominant pinning centers are normal point pinning centers as compatible with the former discussion. Meanwhile, another feature revealed in Figs. 4(a) and 4(b) is a deviation starts to take place at the $\mu_{0} H_{\max }$ (e.g., $\mu_{0} H_{\max }=5.16 \mathrm{~T}$ for $\mathrm{S} 2$ and $3.13 \mathrm{~T}$ for $\mathrm{S} 1$-quenched at $T=15 \mathrm{~K}$ ) and it is much more pronounced for $\mathrm{S} 2$ than $\mathrm{S} 1$-quenched sample. Similar deviations were also observed in $\mathrm{YBa}_{2} \mathrm{Cu}_{3} \mathrm{O}_{7-\delta}$ single crystals and they were attributed to the effect of flux creep. ${ }^{35,39,40}$ This indicates that the flux creep may appear and becomes dominant under high fields at evaluated temperatures in the S2 sample, which presumes the lower position of the peak in Fig. 2(b). ${ }^{41}$

In conclusion, we have performed a comparative study of the $J_{c}\left(\mu_{0} H\right)$ and the vortex pinning among pure and Mn doped $\mathrm{K}_{\mathrm{x}} \mathrm{Fe}_{2-\mathrm{y}} \mathrm{Se}_{2}$ single crystals. The $J_{c}\left(\mu_{0} H\right)$ values can be greatly improved by $\mathrm{Mn}$ doping and post-quenching treatment when comparing to pristine pure sample. Especially, the $1 \%$ and $2 \% \mathrm{Mn}$ doped samples exhibit an anomalous SMP effect for $H \| a b$, as may be attributed to the nonsuperconducting K-Mn-Se inclusions. Two scaling analyses of $F \propto \mu_{0} H$ on $\mathrm{S} 2$ and $\mathrm{S} 1$-quenched crystals provide a solid evidence for the dominance of the normal core point pinning regime, probably caused by the variations of $\mathrm{K}$ atoms. Additionally, the large normal K-Mn-Se inclusions have been assigned to partially induce the normal surface pinning regime in $\mathrm{S} 2$ sample. 
This work was supported by the National Natural Science Foundation of China (No. 11074163) and Science and Technology Commission of Shanghai Municipality (No. 11DZ1100304). W.-L.Y acknowledges support by the National Natural Science Foundation of China (No. 11474211) and the Natural Science Foundation of Jiangsu Province of China (No. BK20141190). M.T.L acknowledges the support of facilities for performing the samples' characterizations during his study in MPI-FKF at Stuttgart.

${ }^{1}$ Y. Kamihara, T. Watanabe, M. Hirano, and H. Hosono, J. Am. Chem. Soc. 130, 3296 (2008).

${ }^{2}$ G. R. Stewart, Rev. Mod. Phys. 83, 1589 (2011).

${ }^{3}$ A. Gurevich, Annu. Rev. Condens. Matter Phys. 5, 35 (2014).

${ }^{4}$ H. Yang, H. Q. Luo, Z. S. Wang, and H. H. Wen, Appl. Phys. Lett. 93, 142506 (2008).

${ }^{5}$ D. L. Sun, Y. Liu, and C. T. Lin, Phys. Rev. B 80, 144515 (2009).

${ }^{6}$ X. L. Wang, S. R. Ghorbani, S. Ik. Lee, S. X. Dou, C. T. Lin, T. H. Johansen, K. H. Müller, Z. X. Cheng, G. Peleckis, M. Shabazi, A. J. Qviller, V. V. Yurchenko, G. L. Sun, and D. L. Sun, Phys. Rev. B 82 , 024525 (2010).

${ }^{7}$ K. J. Kihlstrom, L. Fang, Y. Jia, B. Shen, A. E. Koshelev, U. Welp, G. W. Crabtree, W.-K. Kwok, A. Kayani, S. F. Zhu, and H. H. Wen, Appl. Phys. Lett. 103, 202601 (2013).

${ }^{8}$ E. Dagotto, Rev. Mod. Phys. 85, 849 (2013) and references therein.

${ }^{9}$ Y. Mizuguchi, H. Takeya, Y. Kawasaki, T. Ozaki, S. Tsuda, T. Yamaguchi, and Y. Takano, Appl. Phys. Lett. 98, 042511 (2011).

${ }^{10}$ E. D. Mun, M. M. Altarawneh, C. H. Mielke, V. S. Zapf, R. Hu, S. L. Bud'ko, and P. C. Canfield, Phys. Rev. B 83, 100514(R) (2011).

${ }^{11}$ V. A. Gasparov, A. Audouard, L. Drigo, A. I. Rodigin, C. T. Lin, W. P. Liu, M. Zhang, A. F. Wang, X. H. Chen, H. S. Jeevan, J. Maiwald, and P. Gegenwart, Phys. Rev. B 87, 094508 (2013).

${ }^{12}$ L. Jiao, Y. Kohama, J. L. Zhang, H. D. Wang, B. Maiorov, F. F. Balakirev, Y. Chen, L. N. Wang, T. Shang, M. H. Fang, and H. Q. Yuan, Phys. Rev. B 85, 064513 (2012).

${ }^{13}$ Z. W. Wang, Z. Wang, Y. J. Song, C. Ma, Y. Cai, Z. Chen, H. F. Tian, H. X. Yang, G. F. Chen, and J. Q. Li, J. Phys. Chem. C 116, 17847 (2012).

${ }^{14}$ Y. Liu, Q. Xing, K. W. Dennis, R. W. McCallum, and T. A. Lograsso, Phys. Rev. B 86, 144507 (2012).

${ }^{15}$ X. X. Ding, D. L. Fang, Z. Y. Wang, H. Yang, J. Z. Liu, Q. Deng, G. B. Ma, C. Meng, Y. H. Hu, and H. H. Wen, Nat. Commun. 4, 1897 (2013).

${ }^{16}$ S. V. Carr, D. Louca, J. Siewenie, Q. Huang, A. F. Wang, X. H. Chen, and P. C. Dai, Phys. Rev. B 89, 134509 (2014) and references therein.

${ }^{17}$ H. C. Lei and C. Petrovic, Phys. Rev. B 83, 184504 (2011).
${ }^{18}$ M. T. Li, L. Chen, Z. W. Li, G. H. Ryu, C. T. Lin, and J. C. Zhang, J. Phys.: Condens. Matter 25, 335701 (2013).

${ }^{19}$ J. Y. Ge, J. Gutierrez, M. T. Li, J. C. Zhang, and V. V. Moshchalkov, Appl. Phys. Lett. 103, 052602 (2013).

${ }^{20}$ Z. S. Gao, Y. P. Qi, L. Wang, C. Yao, D. L. Wang, X. P. Zhang, and Y. W. Ma, Physica C 492, 18 (2013).

${ }^{21}$ V. Tsurkan, J. Deisenhofer, A. Günther, H.-A. Krug von Nidda, S. Widmann, and A. Loidl, Phys. Rev. B 84, 144520 (2011).

${ }^{22}$ W. P. Liu, M. T. Li, and C. T. Lin, J. Supercond. Nov. Magn. 27, 2419-2426 (2014).

${ }^{23}$ H. C. Lei and C. Petrovic, Phys. Rev. B 84, 212502 (2011).

${ }^{24}$ H. C. Lei and C. Petrovic, Phys. Rev. B 84, 052507 (2011).

${ }^{25}$ Y. Sun, T. Taen, Y. Tsuchiya, Q. P. Ding, S. S. Pyon, Z. X. Shi, and T. Tamegai, Appl. Phys. Express 6, 043101 (2013).

${ }^{26}$ M. Bonura, E. Giannini, R. Viennois, and C. Senatore, Phys. Rev. B 85, 134532 (2012).

${ }^{27}$ B. Shen, B. Zeng, G. F. Chen, J. B. He, D. M. Wang, H. Yang, and H. H. Wen, Europhys. Lett. 96, 37010 (2011).

${ }^{28}$ E. M. Gyorgy, R. B. van Dover, K. A. Jackson, L. F. Schneemeyer, and J. V. Waszczak, Appl. Phys. Lett. 55, 283 (1989).

${ }^{29}$ See supplementary material at http://dx.doi.org/10.1063/1.4901902 for details on the temperature evolution of isothermal MHLs and $J_{c}\left(\mu_{0} H\right)$ for the $\mathrm{S} 2$ sample.

${ }^{30}$ D. Dew-Hughes, Philos. Mag. 30, 293 (1974).

${ }^{31}$ E. J. Kramer, J. Appl. Phys. 44, 1360 (1973).

${ }^{32}$ Y. Yeshurun and A. P. Malozemoff, Phys. Rev. Lett. 60, 2202 (1988).

${ }^{33}$ Z. A. Sun, Z. Wang, Y. Cai, Z. W. Wang, H. X. Yang, H. F. Tian, S. L. Wu, R. X. Zhang, C. Ma, B. Zhang, and J. Q. Li, Europhys. Lett. 105, 57002 (2014).

${ }^{34}$ T. T. Zhou, X. L. Chen, J. G. Guo, S. F. Jin, G. Wang, X. F. Lai, T. P. Ying, H. Zhang, S. J. Shen, S. C. Wang, and K. X. Zhu, J. Phys.: Condens. Matter 25, 275701 (2013).

${ }^{35}$ T. Higuchi, S. I. Yoo, and M. Murakami, Phys. Rev. B 59, 1514 (1999).

${ }^{36}$ W. K. Yeoh, B. Gault, X. Y. Cui, C. Zhu, M. P. Moody, L. Li, R. K. Zheng, W. X. Li, X. L. Wang, S. X. Dou, G. L. Sun, C. T. Lin, and S. P. Ringer, Phys. Rev. Lett. 106, 247002 (2011).

${ }^{37}$ W. Li, H. Ding, Z. Li, P. Deng, K. Chang, K. He, S. H. Ji, L. L. Wang, X. C. Ma, J. P. Hu, X. Chen, and Q. K. Xue, Phys. Rev. Lett. 109, 057003 (2012).

${ }^{38}$ T. K. Chen, C. C. Chang, H. H. Chang, A. H. Fang, C. H. Wang, W. H. Chao, C. M. Tseng, Y. C. Lee, Y. R. Wu, M. H. Wen, H. Y. Tang, F. R. Chen, M. J. Wang, M. K. Wu, and D. V. Dyck, Proc. Natl. Acad. Sci. U.S.A. 111, 63 (2014)

${ }^{39}$ L. Klein, E. R. Yacoby, Y. Yeshurun, A. Erb, G. Müller-Vogt, V. Breit, and H. Wühl, Phys. Rev. B 49, 4403(R) (1994).

${ }^{40}$ L. Civale, M. W. McElfresh, A. D. Marwick, F. Holtzberg, C. Feild, J. R. Thompson, and D. K. Christen, Phys. Rev. B 43, 13732(R) (1991).

${ }^{41}$ M. R. Koblischka, M. Muralidhar, and M. Murakami, Appl. Phys. Lett. 73, 2351 (1998) 